\title{
Complete Coefficient Criteria for Five-Dimensional Hopf Bifurcations, with an Application to Economic Dynamics
}

\author{
Christos Douskos and Panagiotis Markellos \\ Faculty of Engineering, University of Patras, 26504 Rion, Greece \\ Correspondence should be addressed to Panagiotis Markellos; p.markellos@des.upatras.gr
}

Received 7 May 2015; Accepted 23 June 2015

Academic Editor: Dibakar Ghosh

Copyright (C) 2015 C. Douskos and P. Markellos. This is an open access article distributed under the Creative Commons Attribution License, which permits unrestricted use, distribution, and reproduction in any medium, provided the original work is properly cited.

Paper presents a complete mathematical characterization of coefficient criteria for five-dimensional Hopf bifurcations and an example of the application of these criteria to a model of economic dynamics. The application illustrates that the proposed criteria are practical and useful in determining the existence or nonexistence of Hopf bifurcations of five-dimensional dynamical systems in entire ranges of the system's parameters.

\section{Introduction}

Hopf bifurcations occur in dynamical systems leading to cyclical fluctuations emerging from equilibrium states, and the Hopf bifurcation theorem is a useful tool to establish the emergence of such fluctuations. The theorem describes conditions to be satisfied by the eigenvalues of the Jacobian matrix of the linearized system around an equilibrium, for cycles to bifurcate from the equilibrium. Although the theorem is of local validity, it provides useful information about the system's potential for oscillatory behaviour for a range of a bifurcation parameter. Interpreting the theorem in terms of conditions to be satisfied by the coefficients of the characteristic polynomial at the equilibrium, that is establishing "coefficient criteria" for Hopf bifurcations, facilitates the detection of cycles generated locally at equilibrium and the application of this detection process globally in entire ranges of the system parameters.

In the field of economic dynamics Hopf bifurcations are of interest for the mathematical modelling of endogenous business cycles. Several authors have used this theorem to study the appearance of business cycles in continuous time economic models. For example, Asada [1] and Asada and Yoshida [2] treated three- and four-dimensional Hopf bifurcations by means of coefficient criteria. In recent years the tendency is to consider higher-dimensional dynamics in macroeconomic modelling (see, e.g. [3]), but complete coefficient criteria for Hopf bifurcations have not been proposed so far for $n$-dimensional systems with $n>4$.

The highest dimensionality for which complete coefficient criteria are presently available is $n=4$, and the relevant criteria were provided by Asada and Yoshida [2]. Liu [4] has provided a general criterion for $n$-dimensional Hopf bifurcations, $n \geq 3$, which however is not complete as it applies only to the so called "simple" Hopf bifurcation. This is a special kind of Hopf bifurcation occurring when all eigenvalues of the Jacobian matrix have negative real parts, except the single pair of imaginary eigenvalues characterizing the bifurcation instance, and the simple Hopf bifurcation is therefore related to a change of equilibrium stability.

In this paper we present a complete mathematical characterization of coefficient criteria for five-dimensional Hopf bifurcations, not only for simple Hopf bifurcations. Our proposed criterion for simple Hopf bifurcations is marginally more concise and informative, providing the imaginary eigenvalues and the approximate period of the cycles directly from the coefficients of the characteristic polynomial but is essentially equivalent to Liu's criterion for $n=5$. The main contribution of this paper is the establishment of the coefficient criterion for the existence or nonexistence of nonsimple five-dimensional Hopf bifurcations. We also present an application of our criteria to a model of economic dynamics. The application illustrates that these can be practically and 
usefully employed to determine the existence or nonexistence of Hopf bifurcations of a five-dimensional dynamical system in entire ranges of the system parameters.

The paper is organized into the following sections. Section 2 contains the Hopf bifurcation theorem for $n$ dimensional systems, and Section 3 presents a complete characterization of coefficient criteria for five-dimensional Hopf bifurcations by means of four mathematical propositions covering simple as well as nonsimple Hopf bifurcations. Section 4 contains an application of the proposed criteria to a five-dimensional two-region business cycle model suggested by Asada et al. [5]. In Section 5 we give an example of Hopf bifurcation, established in Section 4, by presenting diagrams illustrating the orbital behaviour of the system associated with the bifurcation. Section 6 contains some concluding remarks. Finally, Appendices A to D contain the proofs of the mathematical propositions.

\section{The Hopf Bifurcation Theorem}

We employ here a version of the Hopf bifurcation theorem from Guckenheimer and Holmes [6] with minor changes in notation. Consider the $n$-dimensional dynamical system:

$$
\dot{x}=f(x, \varepsilon), \quad x \in R^{n}, f \in C^{\infty},
$$

with $\varepsilon \in R$ a bifurcation parameter.

Theorem 1. Suppose that the dynamical system (1) has the following properties.

(I) The system has a smooth curve of equilibria:

$$
f\left(x^{*}(\varepsilon), \varepsilon\right)=0 .
$$

(II) The characteristic equation

$$
\left|\lambda I-D f\left(x^{*}\left(\varepsilon_{c}\right), \varepsilon_{c}\right)\right|=0,
$$

where $D f\left(x^{*}\left(\varepsilon_{c}\right), \varepsilon_{c}\right)$ is the Jacobian matrix of the system at $\left(x^{*}\left(\varepsilon_{c}\right), \varepsilon_{c}\right)$, has a pair of imaginary roots,

$$
\left(\lambda\left(\varepsilon_{c}\right), \bar{\lambda}\left(\varepsilon_{c}\right)\right)=(i \sqrt{\theta},-i \sqrt{\theta}), \quad \theta>0,
$$

and no other roots with zero real parts.

(III) The real part $\operatorname{Re}(\lambda(\varepsilon))$ of $\lambda(\varepsilon)$ satisfies

$$
\left.\frac{d \operatorname{Re}(\lambda(\varepsilon))}{d \varepsilon}\right|_{\varepsilon=\varepsilon_{c}} \neq 0 .
$$

Then, there exists a continuous function $\varepsilon(\gamma)$ with $\varepsilon(0)=$ $\varepsilon_{c}$, and for sufficiently small values of $\gamma \neq 0$ there exists a continuous family of nonconstant periodic solutions $x(t, \gamma)$ of the dynamical system. As $\gamma \rightarrow 0$, the family of periodic solutions collapses at the equilibrium point $x^{*}\left(\varepsilon_{c}\right)$, with their period tending to

$$
T=\frac{2 \pi}{\operatorname{Im}\left(\lambda\left(\varepsilon_{c}\right)\right)}=\frac{2 \pi}{\sqrt{\theta}} .
$$

In the present case $n=5$ the Jacobian matrix of the system is

$$
\begin{aligned}
& J(\varepsilon)=D f\left(x^{*}(\varepsilon), \varepsilon\right)=\left(f_{i j}\right), \\
& f_{i j}=\frac{\partial f_{i}}{\partial x_{j}}, i, j=1, \ldots, 5,
\end{aligned}
$$

with characteristic equation:

$$
\begin{aligned}
|\lambda-J(\varepsilon)| & =p(\lambda) \\
& =\lambda^{5}+b_{1} \lambda^{4}+b_{2} \lambda^{3}+b_{3} \lambda^{2}+b_{4} \lambda+b_{5}=0,
\end{aligned}
$$

where the coefficients $b_{i} \in R,(i=1, \ldots, 5)$ depend on the parameter $\varepsilon$. The aim of this paper is to establish conditions that must be satisfied by these five coefficients for a Hopf bifurcation to occur at the critical value $\varepsilon_{c}$ of the bifurcation parameter $\varepsilon$.

\section{Coefficient Criteria for $n=5$}

The following propositions provide the complete characterization of the Hopf bifurcation in the case of five-dimensional dynamical systems. Proofs of these propositions are provided in Appendices A to D.

Lemma 2. The polynomial $p(\lambda)$ has exactly one pair of imaginary roots $\lambda_{1,2}= \pm i \sqrt{\theta}, \theta>0$, if and only if one of the following sets of conditions, coefficient criteria, is satisfied:

$$
\begin{aligned}
& \left(C_{1}\right) \Phi=\left(b_{3}-b_{1} b_{2}\right)\left(b_{5} b_{2}-b_{3} b_{4}\right)-\left(b_{5}-b_{1} b_{4}\right)^{2}=0, \text { with } \theta= \\
& \left(b_{5}-b_{1} b_{4}\right) /\left(b_{3}-b_{1} b_{2}\right)>0, \\
& \left(C_{2}\right) b_{5}=b_{1} b_{4}, b_{3}=b_{1} b_{2}, \text { and } b_{4}<0 \text {, with } \theta=(1 / 2)\left(b_{2}+\right. \\
& \left.\sqrt{b_{2}^{2}-4 b_{4}}\right)>0, \\
& \left(C_{3}\right) b_{5}=b_{1} b_{4}, b_{3}=b_{1} b_{2}, \text { and } b_{4}=0, b_{2}>0 \text {, with } \theta= \\
& b_{2}>0 .
\end{aligned}
$$

Theorem 3. The polynomial $p(\lambda)$ has one pair of imaginary roots $\lambda_{1,2}= \pm i \sqrt{\theta}, \theta>0$ and all other roots with nonzero real parts if and only if one of the following coefficient criteria is satisfied:

$$
\begin{aligned}
& \left(D_{1}\right):\left\{\left(C_{1}\right), b_{3}-b_{1} \theta_{1} \neq 0\right\}, \\
& \left(D_{2}\right):\left\{\left(C_{2}\right), b_{3} b_{1} \neq 0\right\} .
\end{aligned}
$$

Theorem 4. The polynomial $p(\lambda)$ has one pair of imaginary roots $\lambda_{1,2}= \pm i \sqrt{\theta}, \theta>0$ and all other roots with negative real parts if and only if the following coefficient criterion is satisfied:

$$
(E):\left\{\left(C_{1}\right), b_{1}>0, b_{3}-b_{1} b_{2}<0, b_{3}-b_{1} \theta>0\right\} .
$$

Theorem 5. Under conditions $\left(D_{1}\right)$ or $(E)$, condition (III) of Theorem 1 is equivalent to

$$
\left.\left(Z_{1}\right)(d \Phi(\varepsilon) / d \varepsilon)\right|_{\varepsilon=\varepsilon_{c}} \neq 0,
$$

and under conditions $\left(D_{2}\right)$ condition (III) of Theorem 1 is equivalent to 


$$
\begin{aligned}
& \left(Z_{2}\right)\left\{b_{1}^{\prime}\left(b_{2}+\sqrt{b_{2}^{2}-4 b_{4}}\right)^{2}+2\left(b_{1} b_{2}^{\prime}-b_{3}^{\prime}\right)\left(b_{2}+\sqrt{b_{2}^{2}-4 b_{4}}\right)-\right. \\
& \left.4\left(b_{1} b_{4}^{\prime}-b_{5}^{\prime}\right)\right\}\left.\right|_{\varepsilon=\varepsilon_{c}} \neq 0,
\end{aligned}
$$

where the derivatives are with respect to the bifurcation parameter $\varepsilon$.

Theorem 3 is a complete characterization of condition (II) of Theorem 1, for nonsimple Hopf bifurcation, in the present case of a five-dimensional system. Theorem 4 regards the special case of simple Hopf bifurcation. The proposed here criterion for the simple five-dimensional Hopf bifurcation is marginally more concise and informative but essentially equivalent to Liu's criterion for $n$-dimensional simple Hopf bifurcations in the present case $n=5$ (see Remark 6 in Appendix C).

Finally, Theorem 5 provides a complete characterization of condition (III) of the Hopf bifurcation theorem in the case of a five-dimensional system. The above Theorems 3 and 5 are entirely new results to the authors' knowledge and complement Theorem 4 providing a complete mathematical characterization of the coefficient criteria for Hopf bifurcations in five-dimensional dynamical systems.

The application in the following section illustrates that the proposed criteria are practical and useful in determining the existence or nonexistence of Hopf bifurcations of five-dimensional dynamical systems in entire ranges of the system's parameters and are therefore useful for the analytical investigation of cyclical behaviour in five-dimensional dynamical systems.

\section{Application to Economic Dynamics}

In this section, we present an application of the coefficient criteria stated in the previous section to a typical model of five-dimensional macroeconomic dynamics. For the application we consider a continuous time version of the Kaldorian two-region discrete time business cycle model proposed by Asada et al. [5].

The continuous time version employed here for the application of the proposed coefficient criteria is described by the following five nonlinear differential equations:

$$
\begin{aligned}
& \dot{Y}_{1}(t)=f_{1}=\alpha_{1}\left[-0.36 Y_{1}(t)+75+F_{1}+\delta H(t)\right], \\
& \dot{K}_{1}(t)=f_{2}=F_{1}, \\
& \dot{Y}_{2}(t)=f_{3}=\alpha_{2}\left[-0.36 Y_{2}(t)+75+F_{2}-\delta H(t)\right], \\
& \dot{K}_{2}(t)=f_{4}=F_{2}, \\
& \dot{M}_{1}(t)=f_{5}=\delta H(t)+\beta\left[r_{1}(t)-r_{2}(t)\right],
\end{aligned}
$$

where dots denote derivatives with respect to time and we have abbreviated

$$
\begin{aligned}
F_{i}(t) & =f\left[Y_{i}(t)\right]-0.3 K_{i}(t)-r_{i}(t), \quad i=1,2, \\
r_{i}(t) & =10\left[Y_{i}(t)\right]^{1 / 4}-M_{i}(t), \quad i=1,2, \\
H(t) & =-0.3 Y_{1}(t)+0.3 Y_{2}(t)+40 .
\end{aligned}
$$

The function $f$ is a particular case of the Kaldorian $S$-shaped direct dependence of the investment function on income given by

$$
f(Y)=\frac{80}{\pi} \arctan \left[\frac{9 \pi}{80}(Y-250)\right]+35 .
$$

The subscript $i(=1,2)$ is the index number of a region and $t$ denotes the time. The price levels are considered fixed and the meanings of the symbols are as follows. $Y_{i}$ is the real regional income, $K_{i}$ is the real physical capital stock, and $M_{i}$ is the nominal money stock. The exchange rate is fixed and the total nominal money supply of the two regions is taken to be fixed at $M_{1}+M_{2}=600$.

The parameters of the model are $\alpha_{i}>0$ the adjustment speed of the goods market of each region, $\beta>0$ the degree of capital mobility, and $\delta$ the degree of interregional trade, $0 \leq$ $\delta \leq 1$. We note that under the specifications and functional forms adopted in the formulation of the system of equations (9), the two regional economies are assumed quite similar; any dissimilarity will be due to the possibly unequal speeds of adjustment $\alpha_{1}, \alpha_{2}$. For a full description of the model and its economic foundations see Asada et al. [5].

The system has a smooth curve of equilibria described by the equilibrium values:

$$
\begin{aligned}
Y_{j}^{*}= & \frac{625}{3}+(-1)^{1+j} \frac{1000 \delta}{3(3+5 \delta)}, \quad j=1,2, \\
K_{j}^{*}= & 1000+\frac{10}{3}\left(f\left(Y_{j}^{*}\right)-5\left[\left(Y_{1}^{*}\right)^{1 / 4}+\left(Y_{2}^{*}\right)^{1 / 4}\right]\right) \\
& +(-1)^{1+j} \frac{\delta}{6 \beta}\left[400-3\left(Y_{1}^{*}-Y_{2}^{*}\right)\right],
\end{aligned}
$$

$$
\begin{aligned}
M_{1}^{*}= & 300+5\left[\left(Y_{1}^{*}\right)^{1 / 4}-\left(Y_{2}^{*}\right)^{1 / 4}\right] \\
& +\frac{\delta}{20 \beta}\left[400-3\left(Y_{1}^{*}-Y_{2}^{*}\right)\right] .
\end{aligned}
$$

The equilibrium values $Y_{1}^{*}$ and $Y_{2}^{*}$ depend on the trade parameter $\delta$ only, while the equilibrium values of the other variables depend on both $\beta$ and $\delta$. All equilibrium values are positive for $\beta>\beta_{m} \cong 0.03$ and $0 \leq \delta \leq 1$.

In this application we first focus our search for Hopf bifurcations by reducing the four-dimensional space of parameters of the model to a series of two-dimensional parameter subspaces of the basic parameters $\delta$ and $\beta$. This is done by assuming that the other two parameters $\alpha_{1}$ and $\alpha_{2}$ take equal values $\alpha_{1}=\alpha_{2}=\alpha$ and considering a succession of values for $\alpha$. Alternatively, we set a plausible value, for example, $\alpha_{1}=1$, for $\alpha_{1}$ and consider a succession of values for $\alpha_{2}$. We then apply our coefficient criteria to each parameter plane $(\delta, \beta)$ by creating appropriate plots representing these criteria graphically.

In applying our criterion $\left(D_{1}\right)$ for nonsimple Hopf bifurcations, these plots include the curve $\Phi=0$ (bold), the curve $h=\left(b_{3}-b_{1} b_{2}\right)\left(b_{5}-b_{1} b_{4}\right)=0$ (thin) representing the boundary of the region (light-shaded) in which $h>0$, and 
if required also the curve $b_{5}=0$. According to our criterion $\left(D_{1}\right)$ Hopf bifurcations occur at the points $(\delta, \beta)$ of the curve $\Phi=0$ provided that these points are inside the region where $h=\left(b_{3}-b_{1} b_{2}\right)\left(b_{5}-b_{1} b_{4}\right)>0$ and correspond to such values of $\delta$ and $\beta$ that the condition $b_{3}-b_{1} \theta_{1} \neq 0$, equivalently $b_{5} \neq 0$, is also satisfied.

In applying our criterion $\left(D_{2}\right)$ for nonsimple Hopf bifurcations, the plots include the curves $\Phi=0$ and $h=0$ as before, and if required also the curves $b_{4}=0, b_{1}=0, b_{3}=0$. According to our criterion $\left(D_{2}\right)$ Hopf bifurcations occur at the points $(\delta, \beta)$ of intersection of the curves $\Phi=0$ and $h=\left(b_{3}-b_{1} b_{2}\right)\left(b_{5}-b_{1} b_{4}\right)=0$ which correspond to such values of $\delta$ and $\beta$ that the condition $b_{4}<0$ is also satisfied with $b_{1} \neq 0, b_{3} \neq 0$.

If a nonsimple Hopf bifurcation appears possible at some point $(\delta, \beta)$ under criterion $\left(D_{1}\right)$ or under criterion $\left(D_{2}\right)$, the bifurcation can be verified by application of the corresponding transversality condition $\left(Z_{1}\right)$ or $\left(Z_{2}\right)$. For this last verification either one of the model parameters $\delta, \beta$ may be tested as the bifurcation parameter of Theorem 1 .

A simple Hopf bifurcation is a special kind of nonsimple Hopf bifurcation. Once a nonsimple Hopf bifurcation at the point $(\delta, \beta)$ has been verified as above under criterion $\left(D_{1}\right)$, computation of the characteristic polynomial at the equilibrium values of the system variables corresponding to this pair of parameter values and checking the conditions $b_{1}>$ $0, b_{3}-b_{1} b_{2}<0, b_{3}-b_{1} \theta_{1}>0$ reveals whether the bifurcation is "simple" or not. This last step, to distinguish between nonsimple and simple Hopf bifurcation, can be performed globally for all points of the bifurcation curve, that is, of those segments of the curve $\Phi=0$ which are inside the region where $h=\left(b_{3}-b_{1} b_{2}\right)\left(b_{5}-b_{1} b_{4}\right)>0$ and correspond to such values of $\delta$ and $\beta$ that the condition $b_{3}-b_{1} \theta_{1} \neq 0$, equivalently $b_{5} \neq 0$, is also satisfied.

Following the above guidelines, we firstly apply our coefficient criteria to several $(\delta, \beta)$ parameter planes corresponding to different values of $\alpha_{1}=\alpha_{2}=\alpha$. Figure 1 shows the results for $\alpha=4,1,0.5$. In the left column diagrams of Figure 1 we see that the curve segments of $\Phi=0$ (bold) do not cross with the curve segments of $h=0$ (thin); therefore Hopf bifurcations do not exist under the coefficient criterion $\left(D_{2}\right)$ in the considered region of the $(\delta, \beta)$ parameter plane. However, the leftmost branch of the curve $\Phi=0$ lies within the region for which $h>0$, and the curve $b_{5}=0$ does not appear, therefore Hopf bifurcations may exist under the coefficient criterion $\left(D_{1}\right)$ in the considered region of the $(\delta, \beta)$ parameter plane at the points of the leftmost branch of the curve $\Phi=0$.

For verification we adopt the parameter $\beta$ of the model as the bifurcation parameter and apply condition $\left(Z_{1}\right)$ globally by computing the relevant derivative at points of this branch of the curve $\Phi=0$. This produces the plot shown in the top left frame of Figure 2 which establishes nonzero values of the derivative and therefore establishes the occurrence of Hopf bifurcations globally at the points of this branch of the curve $\Phi=0$.

In the same computation we determine the values of the quantities $b_{1}, b_{3}-b_{1} b_{2}, b_{5}$, required, on the basis of their signs, to characterize the bifurcations as simple. This produces the plots shown in the remaining frames of Figure 2 which establish that $b_{1}>0, b_{3}-b_{1} b_{2}<0, b_{3}-b_{1} \theta_{1}=b_{5}>0$, on the points of this branch. We conclude that this branch of the curve $\Phi=0$ is a Hopf bifurcation curve and the Hopf bifurcations are simple. Note that this also means that this branch is the boundary of the stability region in the $(\delta, \beta)$ plane as shown in Figure 1 (right column frames: dark shaded stability regions).

Let us now consider a fixed value $\alpha_{1}=1$ for $\alpha_{1}$ and a succession of values for $\alpha_{2}$. In such cases, working as before, we find that the curve segments of $\Phi=0$ may cross with the curve segments of $h=0$, as shown, for example, in the case of $\alpha_{1}=1, \alpha_{2}=0.65$, shown in Figure 3. In this case we have as before the leftmost branch of the curve $\Phi=0$ to be a simple Hopf bifurcation curve (see left diagram of Figure 3), but we also find a crossing of $\Phi=0$ (bold) with $h=0$ (thin) suggesting the possible existence of a nonsimple Hopf bifurcation under our coefficient criterion $\left(D_{2}\right)$. Zooming into the neighborhood of the crossing point we obtain the right diagram of Figure 3 where the region in which $b_{4}<0$ is satisfied is also shown (dark shaded). The crossing occurs at the point $\delta^{*}=0.717196, \beta^{*}=0.726274$, outside this region and we conclude that the crossing does not produce a Hopf bifurcation. In this case the usefulness of the coefficient criterion $\left(D_{2}\right)$ is to establish the nonexistence of a Hopf bifurcation in an entire region of the $(\delta, \beta)$ parameter plane, for example, the region shown in the right diagram of Figure 3.

\section{A Bifurcation Example}

In this section, we give an example of Hopf bifurcation, established in Section 4, by presenting diagrams illustrating the cyclical behaviour of the system associated with the bifurcation. To this purpose we choose a specific value $\delta_{0}=$ 0.59 of the parameter $\delta$ and consider the other parameter $\beta$ as the bifurcation parameter of the Hopf bifurcation theorem. The critical value of the bifurcation parameter is $\beta_{c} \cong$ 2.01281996 at which value $\Phi=0$. The point $\left(\delta_{0}, \beta_{c}\right)$ is a point on the simple Hopf bifurcation curve established in the previous section and shown in the middle row diagrams of Figure 1 as the leftmost branch of the curve $\Phi=0$.

Employing numerical integration for a value of $\beta$ slightly smaller than the critical value $\beta_{c}$ and initial values of the system variables very near the equilibrium values, we obtain the time paths of the system variables, as shown for two of the five variables $Y_{1}$ and $Y_{2}$ in the top row of Figure 4. For a certain time interval the system performs oscillations with increasing amplitude until it reaches a time when the values increase rapidly and the system approaches quickly a large stable periodic orbit not related to the Hopf bifurcation.

The "projection" of the five-dimensional orbit in the $\left(Y_{1}, Y_{2}\right)$ plane is shown in the bottom left diagram of Figure 4, while the bottom right diagram shows the orbit near the equilibrium (denoted by a dot). Numerical integration with initial values sufficiently nearer to the equilibrium values produces an orbit converging to the equilibrium. It is apparent that we have a case of subcritical simple Hopf bifurcation in which the equilibrium loses stability because a repelling 

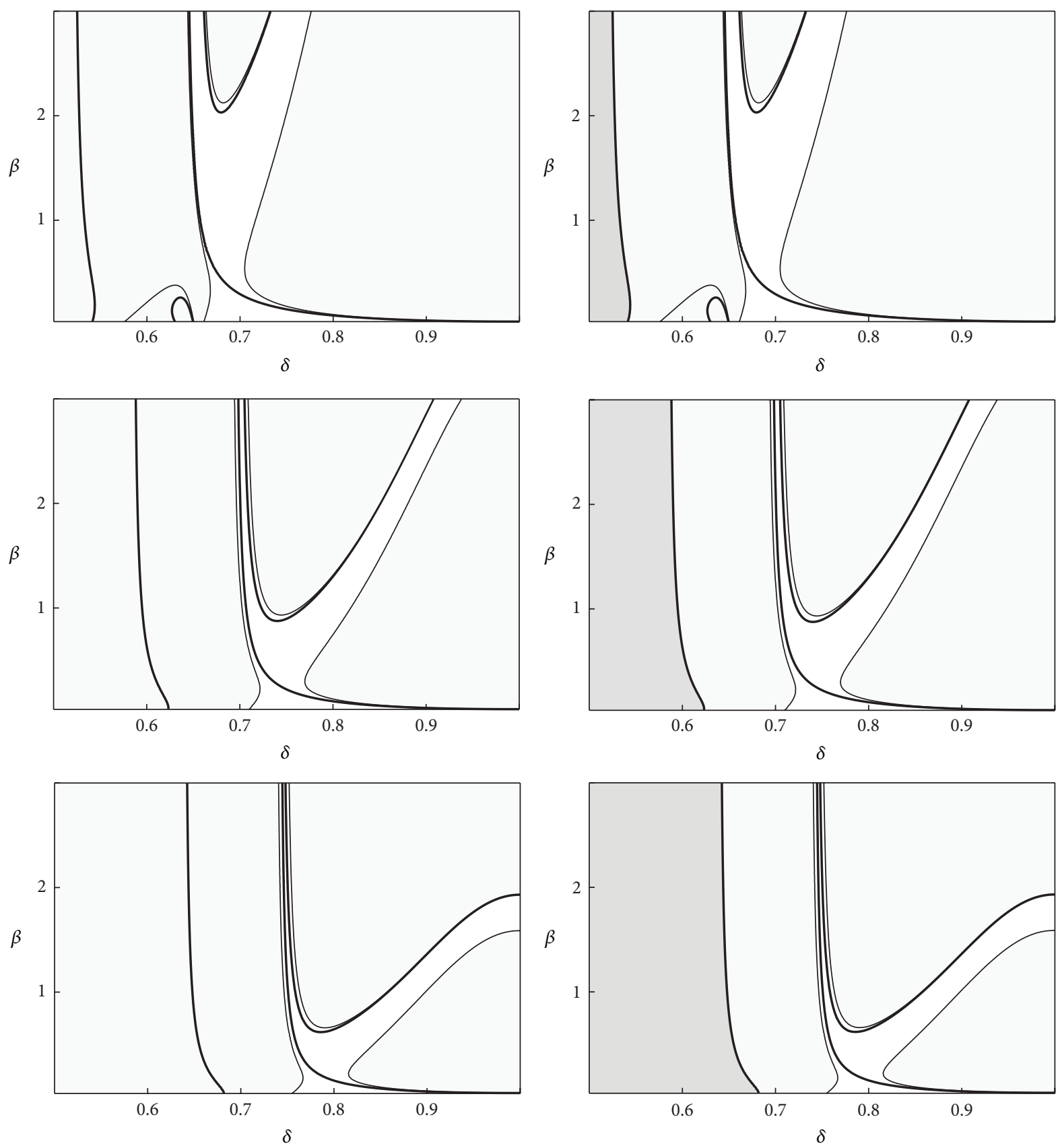

FIgURE 1: Plots of the curves $\Phi=0$ (bold), $h=0$ (thin). Stability regions are dark shaded. Regions with $h>0$ are light shaded. Top to bottom: $\alpha_{1}=\alpha_{2}=4,1,0.5$.

cycle collapses on it. This is a local bifurcation process. Inside the stability region the stable equilibrium coexists with the repelling cycle of the Hopf bifurcation, but in the present case an attracting outer large cycle is also present. Figure 5 shows a schematic representation of the repelling cycles of the present Hopf bifurcation regarding the relevant values $\delta, \beta$ in the parameter plane (left diagram) and two such cycles in actual size in their $\left(Y_{1}, Y_{2}\right)$ plane projections (right diagram) obtained approximately by numerical experiments. For discussions on the two kinds of simple Hopf bifurcation (supercritical and subcritical) and related issues of "corridor stability" and "hysteresis" effects in economic models we refer to Kind [7] and references therein.

\section{Concluding Remarks}

Interpreting the Hopf bifurcation theorem in terms of conditions to be satisfied by the coefficients of the characteristic polynomial at the equilibrium, that is establishing "coefficient criteria" for Hopf bifurcations, facilitates the detection of cycles generated locally at the equilibrium and makes feasible the application of this detection process globally, in the space 

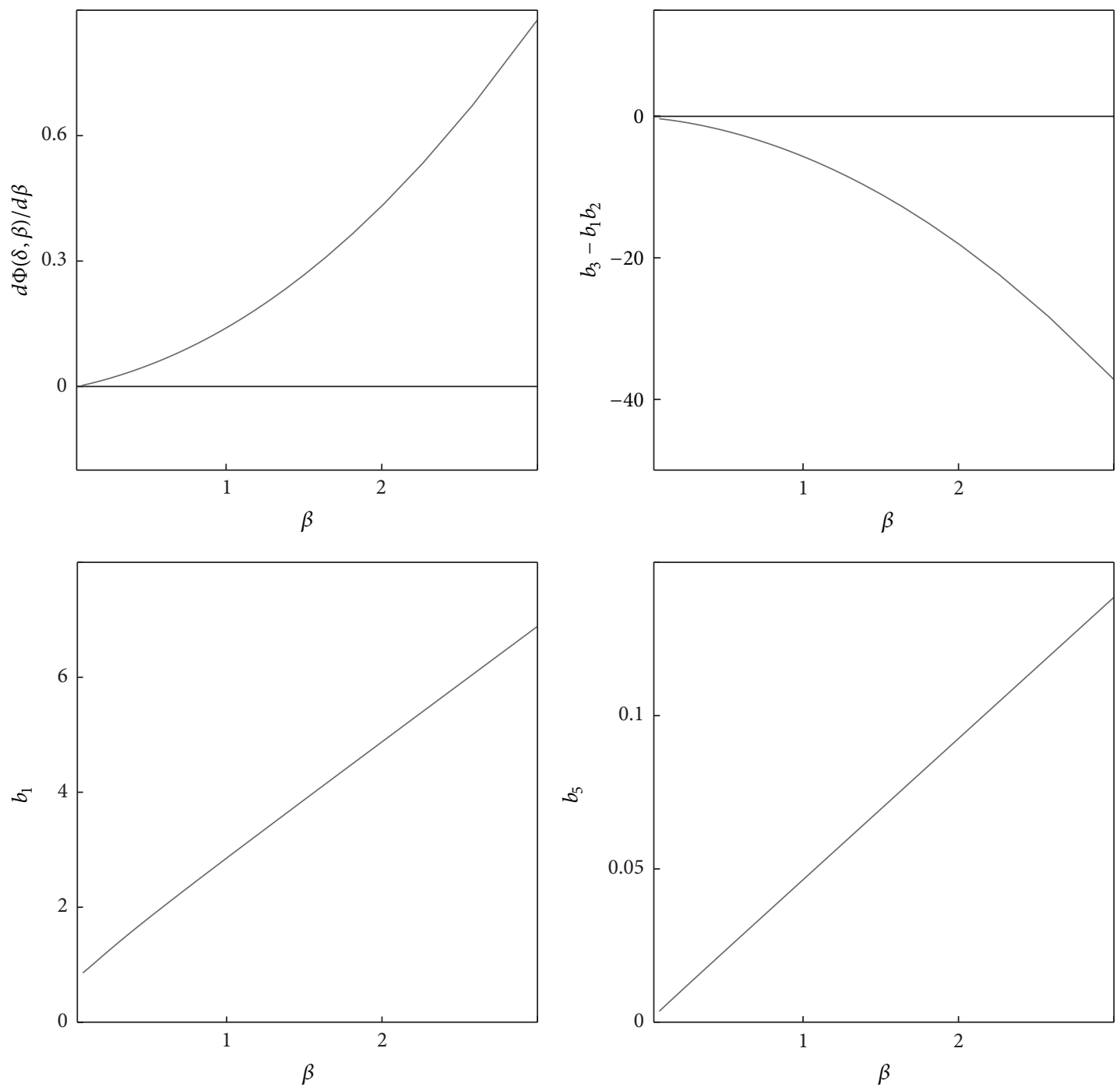

Figure 2: Plots of quantities related to the coefficient criteria $\left(Z_{1}\right)$ and $(E)$ for points $(\delta, \beta)$ on the Hopf bifurcation curve.
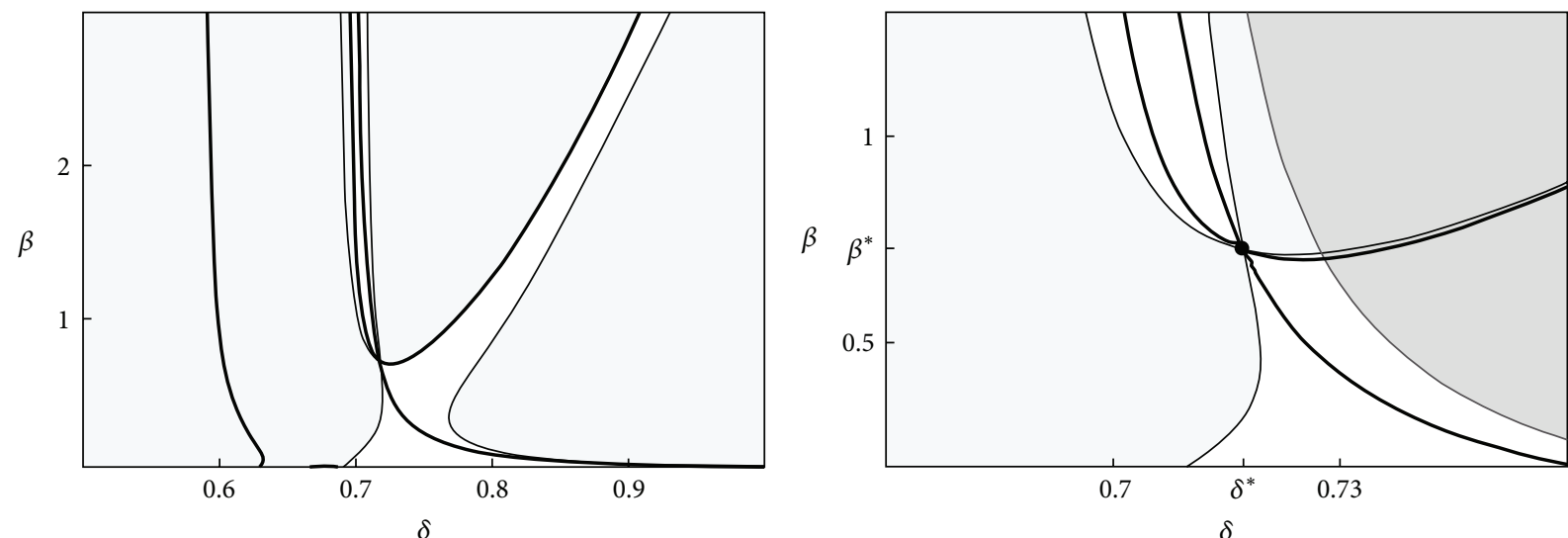

Figure 3: Plots of the curves $\Phi=0$ (bold), $h=0$ (thin) for $\alpha_{1}=1, \alpha_{2}=0.65$. Regions with $h>0$ are light shaded. Region with $b_{4}<0$ is dark shaded. 

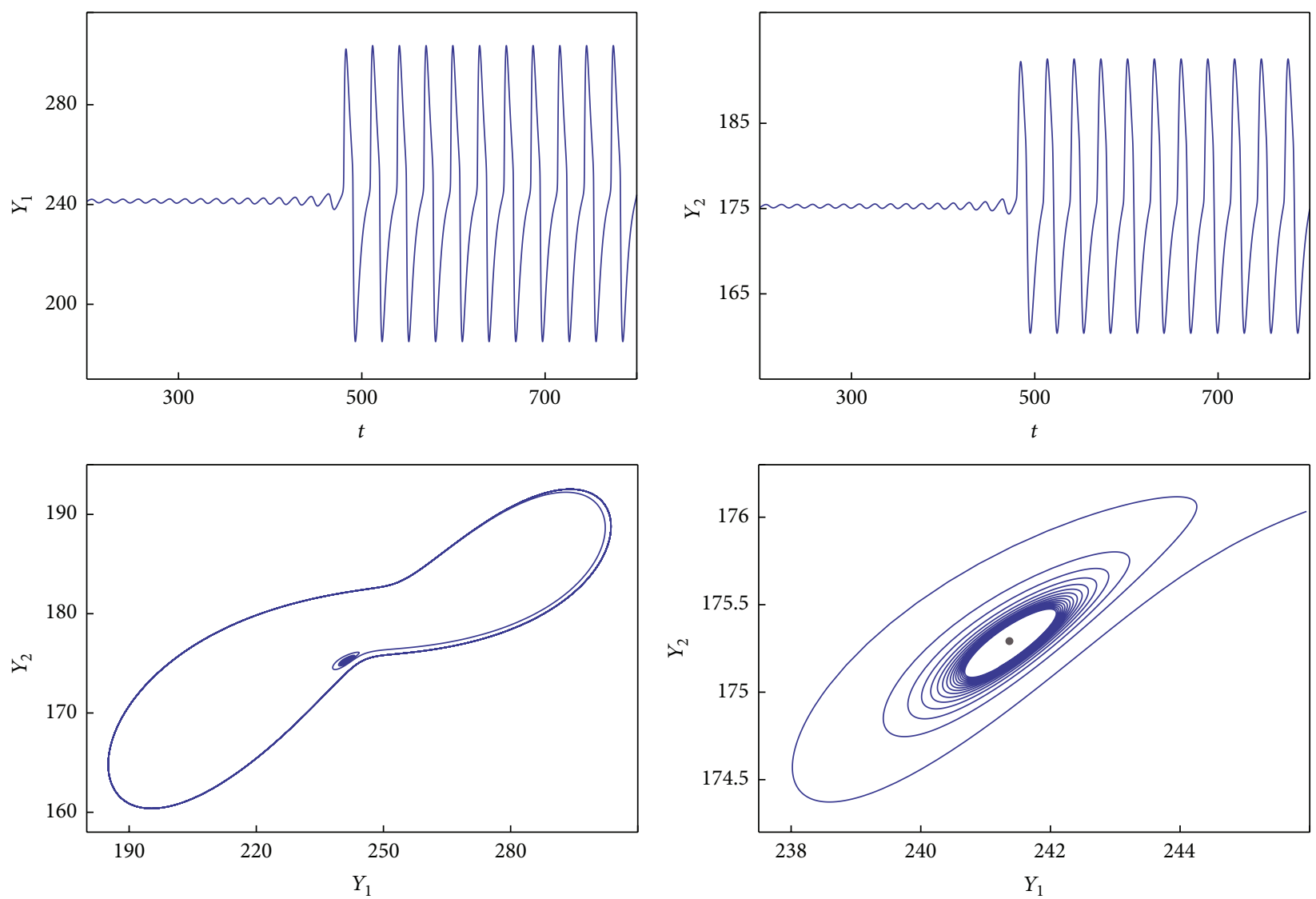

FIGURE 4: Numerical simulation of an orbit starting near the repelling cycle of the subcritical Hopf bifurcation.
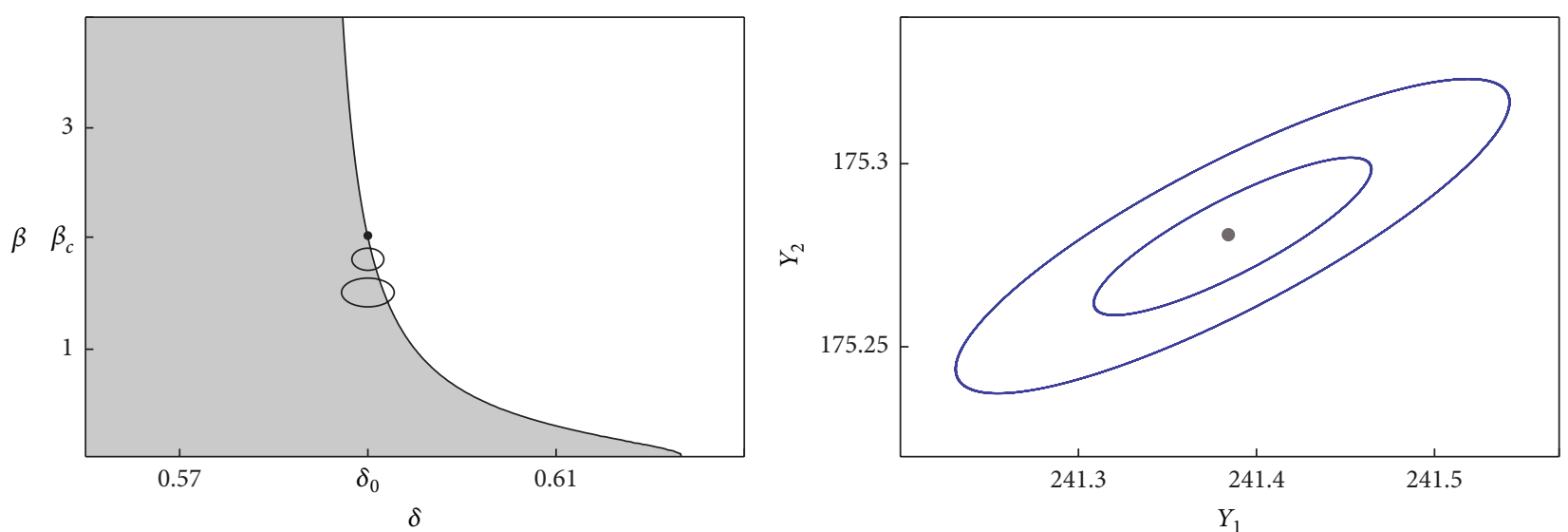

FIGURE 5: Schematic representation of the repelling cycles of the Hopf bifurcation regarding the relevant values $\delta, \beta$ in the parameter plane (left) and approximate representation of two such cycles in actual size in their $\left(Y_{1}, Y_{2}\right)$ plane projections (right).

of the system's parameters, to acquire preliminary information about the system's potential for oscillatory behaviour.

In this paper, we presented a complete mathematical characterization of coefficient criteria for five-dimensional Hopf bifurcations. The criterion proposed here for simple Hopf bifurcations is marginally more concise and informative (see relations (C.7) in Appendix C), providing also the imaginary roots $\pm i \sqrt{\theta}$ of the characteristic polynomial and the approximate period $T=2 \pi / \sqrt{\theta}$ of the cycles directly from the coefficients, but is essentially equivalent to Liu's criterion for $n=5$. The main contribution of this paper is however the derivation of the coefficient criterion for establishing the existence or nonexistence of nonsimple five-dimensional Hopf bifurcations. 
We also suggested a possible manner of conducting the application of our coefficient criteria by presenting an application to a model of nonlinear macroeconomic dynamics. The application showed that our criteria are practical and useful in determining the existence or nonexistence of Hopf bifurcations of five-dimensional dynamical systems in entire ranges of the system's parameters. Finally we presented an example of a bifurcation detected by using our criteria, which turned out to be a subcritical simple Hopf bifurcation, and illustrated by numerical simulation the cyclical behaviour of the system variables due to the repelling cycle associated with the subcritical bifurcation.

\section{Appendices}

\section{A. Proof of Lemma 2}

It is well known that the polynomial

$$
\begin{array}{r}
p(\lambda)=\lambda^{5}+b_{1} \lambda^{4}+b_{2} \lambda^{3}+b_{3} \lambda^{2}+b_{4} \lambda+b_{5}, \\
b_{i} \in R,(i=1, \ldots, 5)
\end{array}
$$

has a pair of imaginary roots $\lambda_{1,2}= \pm i \sqrt{\theta}, \theta>0$, if and only if it can be written in the form

$$
\begin{aligned}
& p(\lambda)=\left(\lambda^{2}+\theta\right) g(\lambda), \\
& \quad \text { with } g(\lambda)=\lambda^{3}+c_{1} \lambda^{2}+c_{2} \lambda+c_{3}, \theta>0,
\end{aligned}
$$

and the multiplication gives

$$
\begin{aligned}
p(\lambda)= & \lambda^{5}+c_{1} \lambda^{4}+\left(c_{2}+\theta\right) \lambda^{3}+\left(c_{3}+c_{1} \theta\right) \lambda^{2} \\
& +c_{2} \theta \lambda+c_{3} \theta .
\end{aligned}
$$

Comparing to (A.1) we obtain

$$
\begin{aligned}
c_{1} & =b_{1}, \\
c_{2}+\theta & =b_{2}, \\
c_{3}+b_{1} \theta & =b_{3}, \\
c_{2} \theta & =b_{4}, \\
c_{3} \theta & =b_{5} .
\end{aligned}
$$

For these relations to be consistent we find that the following equations must be satisfied:

$$
\begin{array}{r}
\theta^{2}-b_{2} \theta+b_{4}=0, \\
b_{1} \theta^{2}-b_{3} \theta+b_{5}=0,
\end{array}
$$

and elimination of $\theta^{2}$ gives

$$
\left(b_{3}-b_{1} b_{2}\right) \theta=b_{5}-b_{1} b_{4} .
$$

The cubic $g(\lambda)$ can be written as

$$
g(\lambda)=\lambda^{3}+b_{1} \lambda^{2}+\left(b_{2}-\theta\right) \lambda+b_{3}-b_{1} \theta,
$$

and we have

$$
\begin{aligned}
p(\lambda)= & \left(\lambda^{2}+\theta\right) g(\lambda) \\
= & \lambda^{5}+b_{1} \lambda^{4}+b_{2} \lambda^{3}+b_{3} \lambda^{2}+\theta\left(b_{2}-\theta\right) \lambda \\
& +\theta\left(b_{3}-b_{1} \theta\right) .
\end{aligned}
$$

A unique positive value of $\theta$ is required for which (A.8) is identical to (A.1).

If $\left(b_{3}-b_{1} b_{2}\right)\left(b_{5}-b_{1} b_{4}\right)<0$, then this requirement cannot be fulfilled as (A.6) gives a negative value of $\theta$. If $\left(b_{3}-b_{1} b_{2}\right)\left(b_{5}-\right.$ $\left.b_{1} b_{4}\right)>0$, then (A.6) gives

$$
\theta=\theta_{1}=\frac{b_{5}-b_{1} b_{4}}{b_{3}-b_{1} b_{2}}>0,
$$

and substituting $\theta_{1}$ in (A.8) we find that (A.8) is identical to (A.1) if and only if

$$
\Phi=\left(b_{3}-b_{1} b_{2}\right)\left(b_{5} b_{2}-b_{3} b_{4}\right)-\left(b_{5}-b_{1} b_{4}\right)^{2}=0,
$$

leading to conditions $C_{1}$.

If one of the quantities $b_{3}-b_{1} b_{2}, b_{5}-b_{1} b_{4}$ is equal to zero, with $\theta>0$, then both these quantities must be equal to zero due to (A.6), and $p(\lambda)$ reduces to

$$
p(\lambda)=\left(\lambda+b_{1}\right)\left(\lambda^{4}+b_{2} \lambda^{2}+b_{4}\right) .
$$

In such case the second of equations (A.5) reduces to $b_{1}\left(\theta^{2}-\right.$ $\left.b_{2} \theta+b_{4}\right)=0$, and (A.5) yield the roots:

$$
\begin{aligned}
& \theta_{2}=\frac{1}{2}\left(b_{2}+\sqrt{b_{2}^{2}-4 b_{4}}\right), \\
& \theta_{3}=\frac{1}{2}\left(b_{2}-\sqrt{b_{2}^{2}-4 b_{4}}\right) .
\end{aligned}
$$

It is required that only one of these roots be positive, and this is true if and only if

$$
\begin{aligned}
& b_{4}<0, \quad \text { or } \\
& b_{4}=0, b_{2}>0 .
\end{aligned}
$$

If $b_{4}<0$, then the two roots (A.12) are

$$
\begin{aligned}
& \theta_{2}=\frac{1}{2}\left(b_{2}+\sqrt{b_{2}^{2}+4\left|b_{4}\right|}\right)>0, \\
& \theta_{3}=\frac{1}{2}\left(b_{2}-\sqrt{b_{2}^{2}+4\left|b_{4}\right|}\right)<0 .
\end{aligned}
$$

If $b_{4}=0, b_{2}>0$, then (A.11) reduces to

$$
p(\lambda)=\left(\lambda+b_{1}\right) \lambda^{2}\left(\lambda^{2}+b_{2}\right),
$$

and the two roots (A.12) are

$$
\begin{aligned}
& \theta_{2}=b_{2}>0, \\
& \theta_{3}=0 .
\end{aligned}
$$


In both these cases there exists exactly one positive value of $\theta$ for which (A.8) is identical to (A.1) as required. Substituting the admissible value $\theta_{2}$ for $\theta$ in (A.8) we find that, due to $b_{3}-$ $b_{1} b_{2}=b_{5}-b_{1} b_{4}=0$, (A.8) is indeed identical to (A.1). This leads in the first case to conditions $C_{2}$ and in the second case to conditions $C_{3}$. In both these cases $\Phi=0$ is also satisfied.

Note that if $b_{4}=0, b_{2} \leq 0$, then none of the roots (A.12) is positive. Also, since the product of the two roots (A.12) is $\theta_{2} \theta_{3}=b_{4}$, it follows that if these roots are real and $b_{4}>0$, then these two roots have the same sign and no unique positive value of $\theta$ exists for which (A.8) would be identical to (A.1). This completes the proof of Lemma 2.

\section{B. Proof of Theorem 3}

Under the conditions $\left(C_{1}\right)$ or $\left(C_{2}\right)$ or $\left(C_{3}\right)$ of Lemma 2 the polynomial $p(\lambda)$ (A.8) has exactly one pair of imaginary roots. This means that any other roots which happen to be complex have nonzero real parts. It remains therefore to determine the conditions under which the polynomial

$$
\frac{p(\lambda)}{\lambda^{2}+\theta}=g(\lambda)=\lambda^{3}+b_{1} \lambda^{2}+\left(b_{2}-\theta\right) \lambda+b_{3}-b_{1} \theta
$$

does not have zero roots. The necessary and sufficient condition for this is obviously that the constant term in (B.1) is nonzero:

$$
b_{3}-b_{1} \theta \neq 0 \text {, }
$$

which is equivalent to $b_{5} \neq 0$ due to (A.8). This is not contained in conditions $C_{1}$ and must be appended to $C_{1}$ (with $\left.\theta=\theta_{1}\right)$.

In the case of conditions $C_{2}$, if $b_{3}, b_{1} \neq 0$ then condition (B.2) is satisfied since $b_{3}-b_{1} b_{2}=0$ and $b_{2} \neq \theta=\left(b_{2}+\right.$ $\left.\sqrt{b_{2}^{2}+4\left|b_{4}\right|}\right) / 2$. But if $b_{3}=b_{1}=0$ then (B.2) is violated. Thus, it is necessary and sufficient to append to $C_{2}$ the condition $b_{3}, b_{1} \neq 0$.

Finally, under conditions $C_{3}$ condition (B.2) cannot be satisfied since $b_{3}-b_{1} b_{2}=0$ with $b_{2}=\theta$, and therefore $b_{3}-$ $b_{1} \theta=0$, and $p(\lambda)$ has zero roots as can be seen from (A.15). This completes the proof of Theorem 3.

\section{Proof of Theorem 4}

The remaining roots of $p(\lambda)$ are the roots of the cubic polynomial (A.7):

$$
\begin{aligned}
g(\lambda) & =\lambda^{3}+c_{1} \lambda^{2}+c_{2} \lambda+c_{3} \\
& =\lambda^{3}+b_{1} \lambda^{2}+\left(b_{2}-\theta\right) \lambda+b_{3}-b_{1} \theta .
\end{aligned}
$$

For this polynomial to have all its roots with negative real parts, it is necessary and sufficient that all primary diagonal determinants of the matrix

$$
\left(\begin{array}{ccc}
c_{1} & c_{3} & 0 \\
1 & c_{2} & 0 \\
0 & c_{1} & c_{3}
\end{array}\right)=\left(\begin{array}{ccc}
b_{1} & b_{3}-b_{1} \theta & 0 \\
1 & b_{2}-\theta & 0 \\
0 & b_{1} & b_{3}-b_{1} \theta
\end{array}\right)
$$

be positive (Routh-Hurwitz conditions for stable roots, cf. [8, pages 221-222]). These determinants are found to be

$$
\begin{aligned}
& \Delta_{1}=b_{1}, \\
& \Delta_{2}=b_{1} b_{2}-b_{3}, \\
& \Delta_{3}=\left(b_{3}-b_{1} \theta\right)\left(b_{1} b_{2}-b_{3}\right),
\end{aligned}
$$

and this leads to conditions

$$
\begin{aligned}
b_{1} & >0, \\
b_{1} b_{2}-b_{3} & >0 \\
b_{3}-b_{1} \theta & >0
\end{aligned}
$$

In the case of $C_{2}$ we have $b_{1} b_{2}-b_{3}=0$ and these conditions are violated. But these conditions are compatible to $C_{1}$, and therefore it is necessary and sufficient to append (C.4) to conditions $C_{1}$, leading to conditions $(E)$. This completes the proof of Theorem 4 .

Remark 6. Note that under conditions $C_{1}$ conditions (C.4) imply that all coefficients of the original polynomial

$$
\begin{aligned}
p(\lambda)= & \lambda^{5}+b_{1} \lambda^{4}+b_{2} \lambda^{3}+b_{3} \lambda^{2}+b_{4}\left\{=\theta\left(b_{2}-\theta\right)\right\} \lambda \\
& +b_{5}\left\{=\theta\left(b_{3}-b_{1} \theta\right)\right\}
\end{aligned}
$$

are positive. Indeed, we have $\theta=\left(b_{5}-b_{1} b_{4}\right) /\left(b_{3}-b_{1} b_{2}\right)>0$, and

$$
\begin{aligned}
& \left\{b_{1}>0, b_{3}-b_{1} \theta>0\right\} \Longrightarrow b_{3}>b_{1} \theta>0, \\
& b_{3}-b_{1} \theta>0 \Longrightarrow b_{5}=\theta\left(b_{3}-b_{1} \theta\right)>0, \\
& \left\{b_{1}>0, b_{3}>0, b_{3}-b_{1} b_{2}<0\right\} \Longrightarrow b_{2}>\frac{b_{3}}{b_{1}}>0, \\
& \left\{b_{3}-b_{1} b_{2}<0, b_{5}>0\right\} \Longrightarrow b_{5}-b_{1} b_{4}<0 \Longrightarrow b_{4}>\frac{b_{5}}{b_{1}} \\
& \quad>0
\end{aligned}
$$

Further, since $\theta>0, b_{4}=\theta\left(b_{2}-\theta\right)>0$, the coefficient of the first power of $\lambda$ in $g(\lambda)$ is also positive: $b_{2}-\theta>0$. Thus all coefficients of (C.1) are positive.

Also, it can be verified that our conditions $(E)$, which can be written in the compact form

(E):

$$
\begin{aligned}
\theta=\frac{b_{5} b_{2}-b_{3} b_{4}}{b_{5}-b_{1} b_{4}}= & \frac{b_{5}-b_{1} b_{4}}{b_{3}-b_{1} b_{2}}>0, \\
& b_{1}>0, b_{3}-b_{1} b_{2}<0, b_{3}-b_{1} \theta>0,
\end{aligned}
$$

are equivalent to the corresponding well-known Liu coefficient conditions (cf. [4]) for simple Hopf bifurcations, which 
in the present case of the quintic characteristic polynomial (A.1) can be written as

$$
\begin{aligned}
& \Delta_{1}=b_{1}>0, \quad \Delta_{2}=\left|\begin{array}{ll}
b_{1} & b_{3} \\
1 & b_{2}
\end{array}\right|>0, \\
& \Delta_{3}=\left|\begin{array}{lll}
b_{1} & b_{3} & b_{5} \\
1 & b_{2} & b_{4} \\
0 & b_{1} & b_{3}
\end{array}\right|>0, \\
& \Delta_{4}=\Phi=\left|\begin{array}{llll}
b_{1} & b_{3} & b_{5} & 0 \\
1 & b_{2} & b_{4} & 0 \\
0 & b_{1} & b_{3} & b_{5} \\
0 & 1 & b_{2} & b_{4}
\end{array}\right|=0, \quad b_{5}>0 .
\end{aligned}
$$

\section{Proof of Theorem 5}

Hopf bifurcations occur under conditions $\left(D_{1}\right)$ or $\left(D_{2}\right)$, and in particular simple Hopf bifurcations occur under conditions $(E)$, provided that condition (III) of Theorem 1 (the transversality condition) is also satisfied.

From the well-known Vieta formulas, giving the coefficients of the polynomial

$$
p(\lambda, \varepsilon)=\lambda^{5}+b_{1} \lambda^{4}+b_{2} \lambda^{3}+b_{3} \lambda^{2}+b_{4} \lambda+b_{5}
$$

from its roots, it follows that the function $\Phi(\varepsilon)$ of conditions $\left(C_{1}\right)$ can be expressed in the form of Orlando's formula:

$$
\begin{aligned}
\Phi(\varepsilon)= & \left(\lambda_{1}+\lambda_{2}\right)\left(\lambda_{1}+\lambda_{3}\right)\left(\lambda_{1}+\lambda_{4}\right)\left(\lambda_{1}+\lambda_{5}\right) \\
& \cdot\left(\lambda_{2}+\lambda_{3}\right)\left(\lambda_{2}+\lambda_{4}\right)\left(\lambda_{2}+\lambda_{5}\right)\left(\lambda_{3}+\lambda_{4}\right) \\
& \cdot\left(\lambda_{3}+\lambda_{5}\right)\left(\lambda_{4}+\lambda_{5}\right) .
\end{aligned}
$$

Assume that the two roots $\lambda_{1,2}$ are

$$
\begin{aligned}
& \lambda_{1,2}=A(\varepsilon) \pm i B(\varepsilon), \\
& \text { with } A\left(\varepsilon_{c}\right)=0, B\left(\varepsilon_{c}\right)=\sqrt{\theta}>0,
\end{aligned}
$$

while $\operatorname{Re}\left(\lambda_{3,4,5}\left(\varepsilon_{c}\right)\right) \neq 0$. Then we have

$$
\begin{aligned}
\Phi(\varepsilon)= & 2 A\left[\left(\lambda_{3}+A\right)^{2}+B\right]\left[\left(\lambda_{4}+A\right)^{2}+B\right] \\
& \cdot\left[\left(\lambda_{5}+A\right)^{2}+B\right]\left(\lambda_{3}+\lambda_{4}\right)\left(\lambda_{3}+\lambda_{5}\right) \\
& \cdot\left(\lambda_{4}+\lambda_{5}\right),
\end{aligned}
$$

and $\Phi\left(\varepsilon_{c}\right)=0$. Differentiating with respect to $\varepsilon$ and putting $\varepsilon=\varepsilon_{c}$ we now obtain

$$
\begin{gathered}
\left.\frac{d \Phi(\varepsilon)}{d \varepsilon}\right|_{\varepsilon=\varepsilon_{c}}=\left\{2\left(B^{2}+\lambda_{3}^{2}\right)\left(B^{2}+\lambda_{4}^{2}\right)\left(B^{2}+\lambda_{5}^{2}\right)\right. \\
\left.\cdot\left(\lambda_{3}+\lambda_{4}\right)\left(\lambda_{3}+\lambda_{5}\right)\left(\lambda_{4}+\lambda_{5}\right) \frac{d A(\varepsilon)}{d \varepsilon}\right\}\left.\right|_{\varepsilon=\varepsilon_{c}} .
\end{gathered}
$$

Under conditions $(E)$ the roots $\lambda_{3}, \lambda_{4}, \lambda_{5}$ have negative real parts at $\varepsilon=\varepsilon_{c}$ and only the last factor in (D.5) may possibly be zero; therefore

$$
\left.\frac{d A(\varepsilon)}{d \varepsilon}\right|_{\varepsilon=\varepsilon_{0}} \neq\left. 0 \Longleftrightarrow \frac{d \Phi(\varepsilon)}{d \varepsilon}\right|_{\varepsilon=\varepsilon_{0}} \neq 0 .
$$

Suppose now that not all three roots $\lambda_{3}, \lambda_{4}, \lambda_{5}$ have negative real parts at $\varepsilon=\varepsilon_{c}$. Then conditions $\left(D_{1}\right)$ or conditions $\left(D_{2}\right)$ apply. Suppose further without loss of generality that $\lambda_{4}\left(\varepsilon_{c}\right)+$ $\lambda_{5}\left(\varepsilon_{c}\right)=0$. Then at $\varepsilon=\varepsilon_{c}$ we have

$$
\begin{aligned}
& \left\{g(\lambda)=\lambda^{3}+b_{1} \lambda^{2}+\left(b_{2}-\theta\right) \lambda+b_{3}-b_{1} \theta\right. \\
& \left.\quad=\left(\lambda^{2}-\lambda_{4}^{2}\right)\left(\lambda-\lambda_{3}\right)=\lambda^{3}-\lambda_{3} \lambda^{2}-\lambda_{4}^{2} \lambda+\lambda_{4}^{2} \lambda_{3}\right\} \\
& \Longrightarrow\left\{b_{1}=-\lambda_{3}, \quad b_{2}-\theta=-\lambda_{4}^{2}, \quad b_{3}-b_{1} \theta=\lambda_{4}^{2} \lambda_{3}\right\} \\
& \Longrightarrow\left\{b_{1}+\lambda_{3}=0, \quad \theta=b_{2}+\lambda_{4}^{2}, \quad b_{3}-b_{1} b_{2}-b_{1} \lambda_{4}^{2}\right. \\
& \left.\quad=\lambda_{4}^{2} \lambda_{3}\right\} \\
& \Longrightarrow b_{3}-b_{1} b_{2}=\lambda_{4}^{2}\left(b_{1}+\lambda_{3}\right)=0 .
\end{aligned}
$$

Since the last of (D.7) violates conditions $\left(D_{1}\right)$, it follows that none of the factors $\left(\lambda_{3}+\lambda_{4}\right),\left(\lambda_{4}+\lambda_{5}\right),\left(\lambda_{4}+\lambda_{5}\right)$ may be zero under conditions $\left(D_{1}\right)$, and therefore (D.6) holds true under conditions $\left(D_{1}\right)$.

We now consider the remaining possible case of Hopf bifurcation, namely $\left(D_{2}\right)$. From the Vieta formulas for the characteristic polynomial (D.1) we obtain

$$
\begin{aligned}
b_{1}= & -2 A-\left(\lambda_{3}+\lambda_{4}+\lambda_{5}\right), \\
b_{2}= & A^{2}+B^{2}+2\left(\lambda_{3}+\lambda_{4}+\lambda_{5}\right) A+\lambda_{3} \lambda_{4}+\lambda_{3} \lambda_{5} \\
& +\lambda_{4} \lambda_{5}, \\
b_{3}= & -\left(A^{2}+B^{2}\right)\left(\lambda_{3}+\lambda_{4}+\lambda_{5}\right) \\
& -2\left(\lambda_{3} \lambda_{4}+\lambda_{3} \lambda_{5}+\lambda_{4} \lambda_{5}\right) A-\lambda_{3} \lambda_{4} \lambda_{5}, \\
b_{4}= & \left(A^{2}+B^{2}\right)\left(\lambda_{3} \lambda_{4}+\lambda_{3} \lambda_{5}+\lambda_{4} \lambda_{5}\right)+2 \lambda_{3} \lambda_{4} \lambda_{5} A, \\
b_{5}= & -\left(A^{2}+B^{2}\right) \lambda_{3} \lambda_{4} \lambda_{5} .
\end{aligned}
$$

Due to the relations $b_{3}\left(\varepsilon_{c}\right)=b_{1}\left(\varepsilon_{c}\right) b_{2}\left(\varepsilon_{c}\right), b_{5}\left(\varepsilon_{c}\right)=b_{1}\left(\varepsilon_{c}\right) b_{4}\left(\varepsilon_{c}\right)$, $b_{4}\left(\varepsilon_{c}\right)<0$, and $B\left(\varepsilon_{c}\right)=\sqrt{\theta_{2}}>0$, of conditions $\left(D_{2}\right)$, at $\varepsilon=\varepsilon_{c}$ the characteristic polynomial takes the form

$$
p\left(\lambda, \varepsilon_{c}\right)=\left(\lambda+b_{1}\right)\left(\lambda^{4}+b_{2} \lambda^{2}+b_{4}\right)
$$

with roots given by

$$
\begin{aligned}
\lambda_{1,2}^{2} & =-\theta_{2}<0, \\
\lambda_{3} & =-b_{1}, \\
\lambda_{4,5}^{2} & =-\theta_{3}>0 .
\end{aligned}
$$


Differentiating now relations (D.8) with respect to $\varepsilon$, putting $\varepsilon=\varepsilon_{c}$, and solving the resulting system of equations for the derivatives of $A, B, \lambda_{3}, \lambda_{4}, \lambda_{5}$, using also relations

$$
\begin{aligned}
& \lambda_{4}\left(\varepsilon_{c}\right)=-\lambda_{5}\left(\varepsilon_{c}\right), \\
& b_{4}\left(\varepsilon_{c}\right)=-\lambda_{4}^{2}\left(\varepsilon_{c}\right) \theta_{2}<0,
\end{aligned}
$$

yields

$$
\begin{aligned}
& \left.\frac{d A(\varepsilon)}{d \varepsilon}\right|_{\varepsilon=\varepsilon_{c}} \\
& =\left.\left\{-\frac{b_{1}^{\prime} \theta_{2}^{2}+\left(b_{1} b_{2}^{\prime}-b_{3}^{\prime}\right) \theta_{2}-\left(b_{1} b_{4}^{\prime}-b_{5}^{\prime}\right)}{2\left(\theta_{2}+b_{1}^{2}\right)\left(\theta_{2}+\lambda_{5}^{2}\right)}\right\}\right|_{\varepsilon=\varepsilon_{c}},
\end{aligned}
$$

which proves that under conditions $\left(D_{2}\right)$ we have

$$
\begin{aligned}
& \left.\frac{d A(\varepsilon)}{d \varepsilon}\right|_{\varepsilon=\varepsilon_{c}} \neq 0 \\
& \left.\Longleftrightarrow\left\{b_{1}^{\prime} \theta_{2}^{2}+\left(b_{1} b_{2}^{\prime}-b_{3}^{\prime}\right) \theta_{2}-\left(b_{1} b_{4}^{\prime}-b_{5}^{\prime}\right)\right\}\right|_{\varepsilon=\varepsilon_{c}}
\end{aligned}
$$$$
\neq 0 \text {. }
$$

Finally, due to the value of $\theta_{2}$, given by the first of (A.12), the last condition can be written in the form

$$
\begin{aligned}
& \left\{b_{1}^{\prime}\left(b_{2}+\sqrt{b_{2}^{2}-4 b_{4}}\right)^{2}\right. \\
& +2\left(b_{1} b_{2}^{\prime}-b_{3}^{\prime}\right)\left(b_{2}+\sqrt{b_{2}^{2}-4 b_{4}}\right) \\
& \left.\quad-4\left(b_{1} b_{4}^{\prime}-b_{5}^{\prime}\right)\right\}\left.\right|_{\varepsilon=\varepsilon_{c}} \neq 0 .
\end{aligned}
$$

This completes the proof of Theorem 5.

\section{Conflict of Interests}

The authors declare that there is no conflict of interests regarding the publication of this paper.

\section{References}

[1] T. Asada, "Kaldorian dynamics in an open economy," Journal of Economics, vol. 62, no. 3, pp. 239-269, 1995.

[2] T. Asada and H. Yoshida, "Coefficient criterion for four-dimensional Hopf bifurcations: a complete mathematical characterization and applications to economic dynamics," Chaos, Solitons \& Fractals, vol. 18, no. 3, pp. 525-536, 2003.

[3] C. Chiarella, P. Flaschel, G. Gong, and W. Semmler, "Nonlinear Phillips curves, complex dynamics and monetary policy in a Keynesian macro model," Chaos, Solitons and Fractals, vol. 18, no. 3, pp. 613-634, 2003.

[4] W. M. Liu, "Criterion of Hopf bifurcations without using eigenvalues," Journal of Mathematical Analysis and Applications, vol. 182, no. 1, pp. 250-256, 1994.

[5] T. Asada, T. Inaba, and T. Misawa, "An interregional dynamic model. The case of fixed exchange rates," Studies in Regional Science, vol. 31, no. 2, pp. 29-41, 2001.
[6] J. Guckenheimer and P. Holmes, Nonlinear Oscillations, Dynamical Systems, and Bifurcations of Vector Fields, Springer, New York, NY, USA, 1983.

[7] C. Kind, "Remarks on the economic interpretation of Hopf bifurcations," Economics Letters, vol. 62, no. 2, pp. 147-154, 1999.

[8] G. Gandolfo, Economic Dynamics, Springer, New York, NY, USA, 1996. 

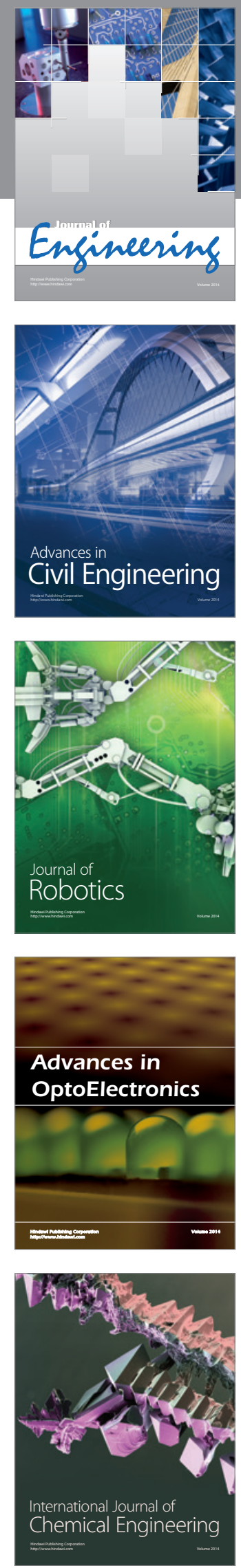

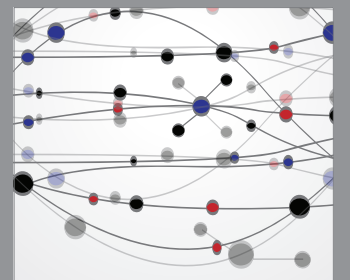

The Scientific World Journal
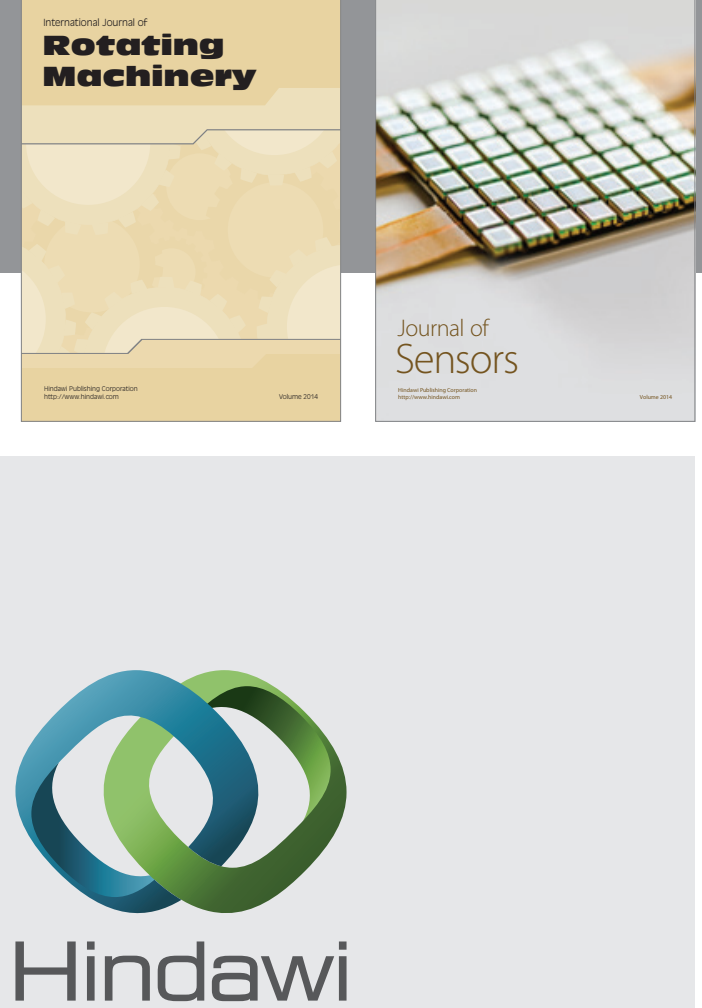

Submit your manuscripts at http://www.hindawi.com
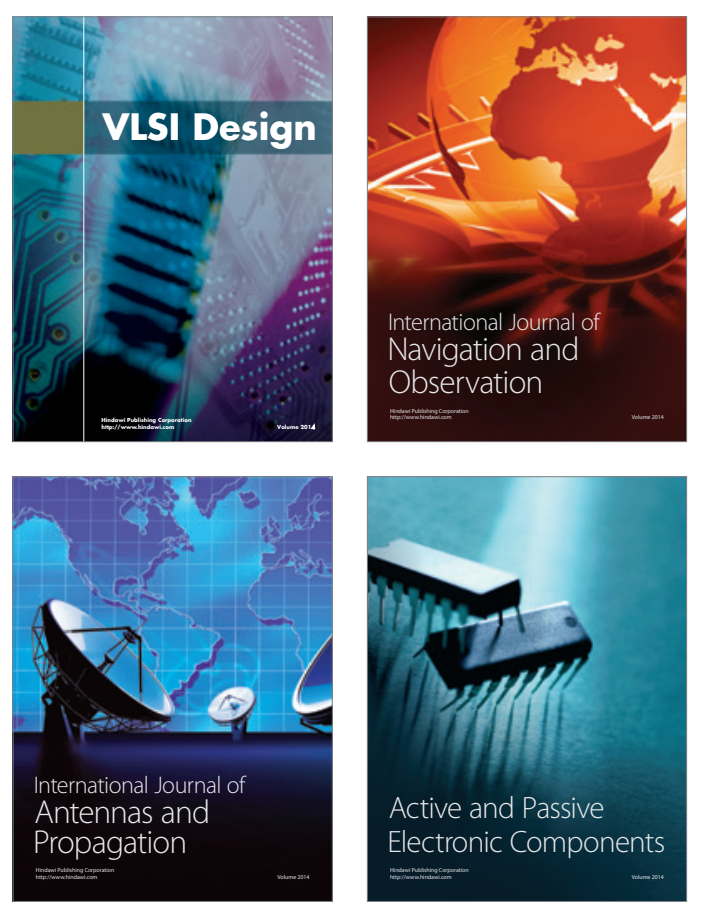
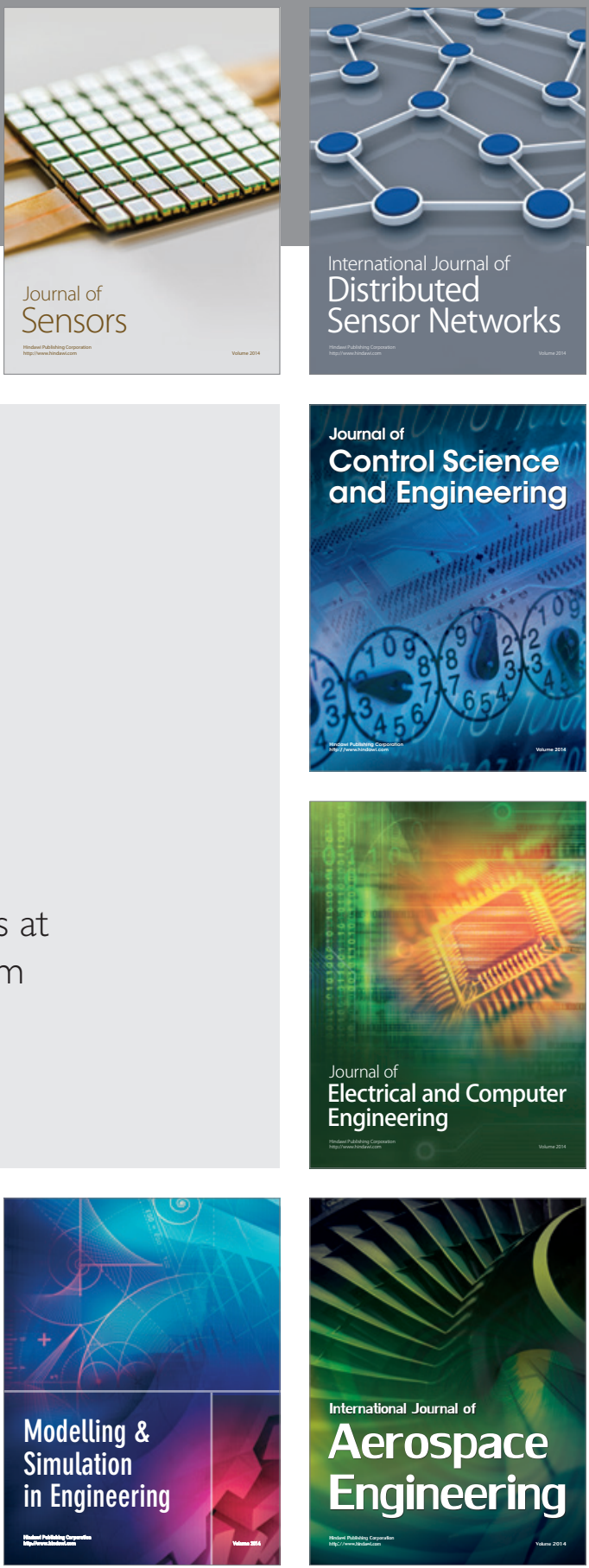

Journal of

Control Science

and Engineering
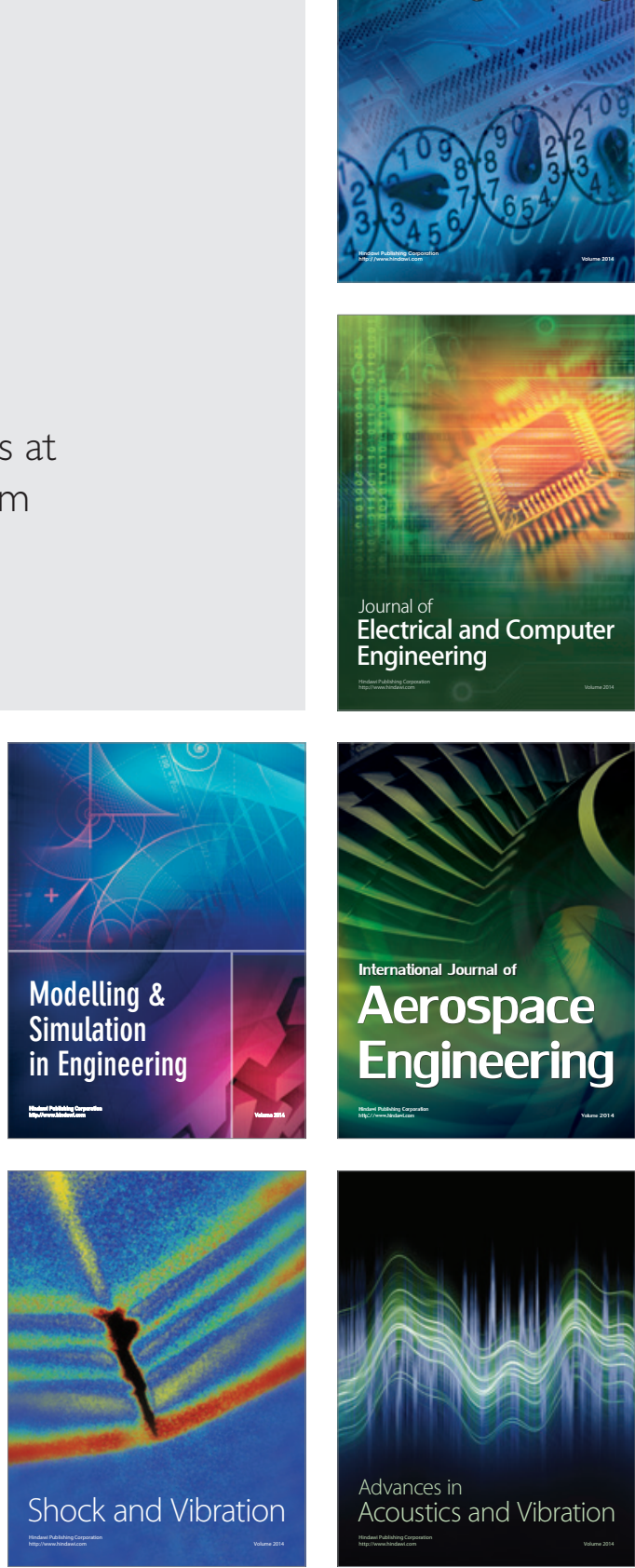